
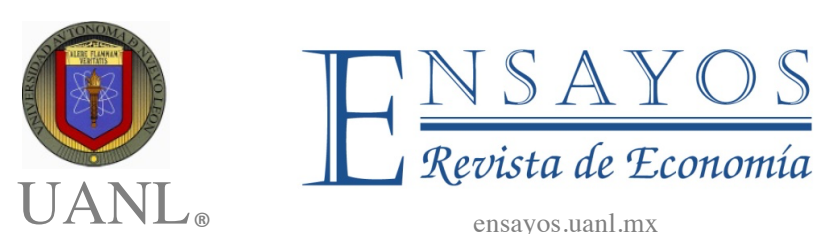

ensayos.uanl.mx

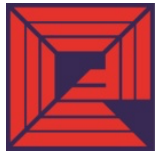

Facultad de Economía

\title{
Crecimiento económico y gasto público en salud según población objetivo en México
}

\section{Economic Growth and Public Spending on Health According to Target Population in Mexico}

Jorge José Luis Reynoso-González* Adrián De León Arias ${ }^{\star *}$

\begin{tabular}{|c|c|}
\hline $\begin{array}{l}\text { Información del } \\
\text { artículo }\end{array}$ & Resumen \\
\hline $\begin{array}{l}\text { Recibido: } \\
16 \text { junio } 2020\end{array}$ & \multirow{9}{*}{$\begin{array}{l}\text { En este artículo, se analiza la relación de largo plazo entre } \\
\text { gasto público en salud (GPS) y crecimiento económico } \\
\text { (CE) en México, desde una perspectiva regional y } \\
\text { considerando el gasto público en salud hacia la población } \\
\text { con y sin acceso a seguridad social. En particular, } \\
\text { ofrecemos un análisis diferencial por tipo de acceso a } \\
\text { servicios de salud y región que enriquece la literatura al } \\
\text { respecto. El análisis econométrico se desarrolla a través de } \\
\text { datos en panel con información de PIB y GPS de } 32 \\
\text { entidades federativas, en el período de } 1993 \text { a } 2017 \text {, y } \\
\text { aplica técnicas de cointegración y modelación mediante } \\
\text { MG-FMOLS. Los principales resultados encuentran } \\
\text { evidencia a favor de una relación de largo plazo entre CE } \\
\text { y ambos tipos de GPS. Adicionalmente, se explora la } \\
\text { relación entre estos resultados y el nivel de desarrollo por } \\
\text { entidad federativa. }\end{array}$} \\
\hline Aceptado: & \\
\hline 16 abril 2021 & \\
\hline $\begin{array}{l}\text { Clasificación JEL: } \\
\text { O47; I18; I14; H55 }\end{array}$ & \\
\hline Palabras clave: & \\
\hline Crecimiento & \\
\hline $\begin{array}{l}\text { Económico; Gasto } \\
\text { público en salud; }\end{array}$ & \\
\hline Seguridad Social; & \\
\hline $\begin{array}{l}\text { Desarrollo económico } \\
\text { regional }\end{array}$ & \\
\hline
\end{tabular}

* Universidad de Guadalajara, jorge.reynoso@academicos.udg.mx; ** Universidad de Guadalajara.

ISSN Electrónico: 2448-8402 | ISSN Impreso: 1870-221X | (C2021 Los autores @) (1) 


\begin{tabular}{|c|c|}
\hline Article information & \multirow{11}{*}{$\begin{array}{l}\text { This article analyzes the long-term relationship between } \\
\text { Public Spending on Health (PHS) and economic growth } \\
\text { (EG) in Mexico through a regional perspective and } \\
\text { considering public spending on health to the population, } \\
\text { with and without access to social security. In particular, we } \\
\text { offer a differential analysis by type of access to health } \\
\text { services and region that enriches the literature in this } \\
\text { regard. The econometric analysis is developed through } \\
\text { panel data with GDP and PHS information from } 32 \text { states } \\
\text { in a period from } 1993 \text { to } 2017 \text { and applies cointegration } \\
\text { and modeling techniques using MG-FMOLS. The main } \\
\text { results find evidence in favor of a long-term relationship } \\
\text { between CE and both types of GPS. Additionally, the } \\
\text { relationship between these results and the level of } \\
\text { development by state is explored. }\end{array}$} \\
\hline Received: & \\
\hline 16 June 2020 & \\
\hline $\begin{array}{l}\text { Accepted: } \\
16 \text { April } 2021\end{array}$ & \\
\hline $\begin{array}{l}\text { JEL Classification: } \\
\text { O47; I18; I14; H55 }\end{array}$ & \\
\hline Keywords: & \\
\hline Economic Growth; & \\
\hline Public expenditure on & \\
\hline Health; Social security; & \\
\hline Regional Economic & \\
\hline Development & \\
\hline
\end{tabular}

\section{Introducción}

El objetivo general de este trabajo es analizar la relación de largo plazo que existe entre el gasto público en salud (GPS) y crecimiento económico (CE). Estudios realizados en el nivel internacional han mostrado la existencia de dicha relación (véase Gerdtham \& Jönsson (2000), Wang (2011), Reeves et al. (2013), y Abdullah et al. (2017)); mientras que investigaciones realizadas para México, sugieren promover políticas orientadas a mejorar la salud, ya que contribuyen al CE (véase, por ejemplo, Mayer-Foulkes $(2001,2008)$ y Lorenzo-Valdes \& Ruiz-Porras (2017)). En este trabajo, en particular, se explora la relación entre GPS y CE de México en una perspectiva intranacional y una diferenciación en el GPS según población objetivo. Lo anterior, para aprovechar la riqueza de información que ofrece el análisis regional, mientras se controla por diferencias tecnológicas, institucionales, culturales y empresariales que se observan entre países. Además, al desagregar el GPS, según población que se atiende, permite incorporar al análisis características socioeconómicas diferentes.

Lo anterior resulta de interés, dado que, en México, el sistema de salud es mixto y fragmentado, en particular, el sector público ofrece servicios de salud a través de instituciones de SS, tales como IMSS, ISSSTE, PEMEX, SEDENA y SEMAR. Y por otro lado, existen instituciones y programas que atienden a población sin SS, tales como la Secretaría de salud, Servicios Estatales de Salud y Seguro Popular ${ }^{1}$ (Gómez Fröde, 2017; Martínez-Trejo, 2018; OCDE, 2016). Es importante hacer distinción entre estos dos tipos de oferta de

1 El Seguro Popular deja de existir el 31 de diciembre del 2019 y es sustituido por el Instituto de Salud para el Bienestar (INSABI). Sin embargo, esto no afecta el análisis de esta investigación debido a que el período abarcado es 1993-2017. 
servicios de salud, específicamente en dos características: 1) el paquete de servicios que ofrecen y 2) la forma de acceso, es decir, contributiva o subsidiada, ya que puede tener diferentes implicaciones para el crecimiento económico.

Respecto a la diferencia en el paquete de servicios, las instituciones de SS ofrecen mayores beneficios en comparación con las instituciones que no son de SS. Por ejemplo, un estudio de la OCDE (2016) revela que mientras el Seguro Popular realiza 319 consultas ambulatorias por cada 1,000 afiliados, el IMSS e ISSSTE efectúan 338 y 620, respectivamente. Más aún, de acuerdo con Grossman (2000), el consumo de productos y servicios de salud es un determinante en la acumulación de salud y, en este sentido, es de esperarse que el GPS desagregado que es dirigido a la población con y sin SS tenga efectos disímiles sobre el CE.

En este contexto, el presente trabajo analiza el gasto público que se destina a la población con y sin SS, y su relación con el CE de México. En particular, para realizar dicho análisis, se implementarán técnicas de series de tiempo adecuadas para paneles de datos, ya que este enfoque permite combinar propiedades de series de tiempo y corte transversal. Adicionalmente, se efectuarán pruebas de raíz unitaria y de cointegración, así como estimación de elasticidades de largo plazo mediante la técnica de Mínimos Cuadrados Ordinarios Completamente Modificados en Medias de Grupo (MG-FMOLS, por sus siglas en inglés), propuesta por Pedroni (2001), además, se analiza causalidad en el sentido de Granger a través de un modelo de Vector de Corrección de Errores en Panel (PVEC, por sus siglas en inglés).

En cuanto a los resultados de las pruebas de cointegración, estos muestran la existencia de movimientos comunes y equilibrio de largo plazo entre CE y ambos tipos de GPS. El principal hallazgo es que, en el nivel nacional, el GPS desagregado por población objetivo tiene efecto positivo de largo plazo sobre el CE; sin embargo, dicho efecto es disímil, siendo mayor el del GPS destinado a la población con SS. En el nivel estatal, se observan diferencias en la productividad del GPS, donde se explora su relación con el nivel de desarrollo de las entidades federativas, tal que el menor nivel de desarrollo se asocia con una elasticidad mayor de ambos tipos de GPS sobre el CE.

Respecto al análisis de causalidad en el sentido de Granger, que podría condicionar los resultados mencionados en el párrafo anterior, nuestros resultados muestran evidencia de efecto de causalidad de largo plazo de los dos GPS, con y sin SS, hacia el PIB; y del PIB hacia el GPS sin SS. Por otro lado, existe efecto causal de corto plazo bidireccional entre el PIB y ambos GPS, con y $\sin$ SS. 
La estructura de este trabajo es como sigue: En la sección 2, se presenta la revisión de literatura sobre $\mathrm{CE}$ y salud, así como estudios empíricos sobre la relación de GPS y CE, en el nivel internacional y para México. En la sección 3 , se presentan los datos y metodología utilizada. En la sección 4, se reportan los resultados. Por último, en la sección 5, se exponen las conclusiones.

\section{Revisión de literatura}

Un área de amplio interés en la literatura sobre crecimiento económico es la identificación y dinámica de los factores que lo determinan. Desde una perspectiva básica, se enfatiza el papel del capital físico en el proceso de producción como el factor fundamental de crecimiento, mientras que estudios basados en el crecimiento endógeno han introducido otros factores en el debate, tales como capital humano, innovación e infraestructura social, entre otros (Romer, 2012). En general, la dinámica de esos factores se da a través de su acumulación en un sector especializado con rendimientos crecientes o, al menos, donde se da una aparición relativamente lenta de rendimientos decrecientes. Dentro de los trabajos desarrollados bajo el enfoque de crecimiento endógeno, la salud es un tema que sobresale como uno de los determinantes que contribuyen al incremento del nivel de producción de los países. La idea central es que mejoras en el estado de salud de la población conducen a un mayor crecimiento económico. Es difícil analizar e incluir la salud en el modelo de Solow, dado que, como lo señala Grimm (2011), es limitado, por ello, solo se realiza a través del incremento en la tasa de crecimiento de la población y como un factor asociado a la calidad, en el desempeño de los trabajadores.

En lo que sigue, se presenta un resumen de resultados de investigación que identifican la importancia de la salud como un factor de crecimiento económico, desde un nivel microeconómico a un nivel más agregado. Un trabajo seminal en esta empresa, es Mushkin (1962) quien sostiene que individuos más sanos son más productivos, tanto como productores como consumidores, y pueden alcanzar niveles de ingreso más altos. Más aún, niveles más altos de ingreso podrían conducir a niveles de salud mayores. La salud como parte del capital humano refleja la calidad de este, y la inversión en dicho componente proporciona productividad dentro de la empresa, lo que facilita una mayor productividad a la economía nacional (Becker, 1962; Schultz, 1961). Por otro lado, Mincer (1981) expone que el deterioro de la salud junto con la obsolescencia de las habilidades es la depreciación del capital humano, aunque no indefinidamente, mediante la producción de salud $\mathrm{y}$ reentrenamiento.

Por su parte, Grossman (2000) también observa que la salud se asocia con mayores niveles de ingreso; en su modelo plantea la salud como un stock de 
capital que produce días saludables, así, los individuos pueden invertir combinando tiempo (por ejemplo, visitas al médico) y, productos y servicios comprados en el mercado (por ejemplo, servicios médicos y medicinas). Asimismo, Bleakley (2010) sostiene que la salud puede afectar el ingreso por medio de dos canales del capital humano, la primera y más directa es que la enfermedad reduce la habilidad de trabajar, y la segunda es que la tasa de utilización del capital humano puede ser mayor, si pasa menos tiempo inactivo debido a la enfermedad.

La salud es un aspecto crucial del capital humano y, por lo tanto, un ingrediente elemental en el crecimiento económico; pues trabajadores que son física y emocionalmente más sanos, son más energéticos y más robustos; son más productivos y tienen ingresos más altos, además de reducir el ausentismo por enfermedad (Bloom, Canning, y Sevilla, 2001). En trabajos como Bloom et al. (2001), y Bloom, Canning y Sevilla (2004), se encontró que la salud tiene un impacto positivo y estadísticamente significativo en el crecimiento económico; el primero sugiere que por cada año que se incremente la esperanza de vida contribuye a un incremento en el producto en un cuatro por ciento.

Bloom et al. (2004) realizan extensa revisión de literatura sobre el efecto que tiene la salud en el crecimiento económico, y encuentran que existe una relación positiva. Por su parte, Acemoglu y Johnson (2007) comentan que la mejora en salud es un objetivo social importante que tiene beneficios directos en términos de alargar y mejorar la vida de millones de personas, además, existe un creciente consenso de que la mejora en la salud puede tener beneficios indirectos, a través del aceleramiento en el crecimiento económico. Sin embargo, la evidencia que da soporte a este consenso no es conclusiva todavía; otros autores, como Barro (1996), encuentran una relación bidireccional entre salud y crecimiento económico, es decir, que al mejorar la salud se produce un mayor crecimiento y que a un mayor nivel de crecimiento habrá mayor acumulación de salud.

Como se puede ver, existe una extensa literatura que aborda el tema de la salud y crecimiento económico con un amplio consenso sobre una relación positiva entre ambos (Grimm, 2011). Aunque la correlación positiva entre la salud y el crecimiento económico está bien establecida, los mecanismos subyacentes son complejos y difíciles de discernir (Bloom, Prettner, y Bloom, 2018). Una estrategia para analizar dichos mecanismos, en la literatura al respecto, es analizar la vinculación de la oferta de salud, vía el gasto público.

Destacando la importancia del gasto en salud, Wang (2011) comenta que mejora la calidad de vida en la población y refleja la intención de desarrollo económico en un país. Este gasto incrementa la seguridad social, tranquilidad y bienestar que incrementa la productividad laboral (Amiri y Ventelou, 2012); además, es aceptado como un indicador de desarrollo económico, pues es un 
factor decisivo para la reducción de pobreza y para la promoción del desarrollo sostenible (Dincer y Yüksel, 2019).

Por otro lado, mejorar la salud a partir del gasto, no solo podría tener efecto directo en la productividad, también podría ser indirectamente, ya que está relacionada de manera positiva con la escolaridad; Grossman (2000) explica que mejoras en la salud podrían conducir a un mayor nivel de escolaridad, y que mayor nivel de escolaridad podría hacer más eficiente la producción de salud.

Con objeto de identificar los aspectos diferenciales de la relación entre gasto en salud y CE, se ha desarrollado una amplia literatura, basada mayormente en análisis de series de tiempo. En seguida, se expone una breve revisión de estudios que examinan dicha relación con datos internacionales.

En un trabajo de amplia referencia, Rivera \& Currais $(1999,2003)$ analizan el efecto del gasto en salud en el ingreso para países de la OCDE, mediante una ecuación log-lineal derivada del modelo de Solow extendido considerando salud, y encuentran un efecto positivo. Sin embargo, cuando advierten de la posible existencia de endogeneidad, los regresores estimados estarían correlacionados con el término de perturbación y la estimación por Mínimos Cuadrados Ordinarios, y serían sesgados e inconsistentes, por tal razón, llevan a cabo pruebas de Hausman para evaluar endogeneidad, lo que los conduce a utilizar variables instrumentales; los resultados sugieren que no se puede rechazar una causación de reversa del ingreso hacia el gasto.

Por su parte, Gerdtham \& Löthgren (2000) examinan la relación de largo plazo entre el gasto en salud y el PIB para 21 países integrantes de la OCDE, para el período de 1960 a 1997, además realizan pruebas de raíz unitaria y de cointegración; sus resultados indican que el gasto en salud y crecimiento en el PIB están cointegrados. De manera similar, Baltagi \& Moscone (2010) exploran la relación de largo plazo entre el PIB y gasto en salud para 20 países miembros de la OCDE en un período de 1971 a 2004. Encuentran que existe cointegración entre estas variables; adicionalmente, estiman la elasticidad del gasto respecto al PIB y encuentran que la salud es un bien necesario y no un bien de lujo.

Con un enfoque de análisis de panel tipo cuantil, Wang (2011) examina el gasto total en salud de 31 países en un período de 1986 a 2007. Implementa pruebas de cointegración y estima el efecto del gasto en salud sobre el PIB, mediante la técnica FMOLS, así como regresión cuantílica. Sus resultados muestran que, en el largo plazo, el GPS mejora el CE, sin embargo, el efecto es diferenciado de acuerdo con el nivel de crecimiento de cada país. 
Utilizando paneles de datos, Reeves, Basu, Mckee, Meissner y Stuckler (2013) utilizan técnicas de panel con efectos fijos con información del PIB y gasto público por sectores de 25 países de la OCDE, en el período de 1995 a 2010; concluyen que el gasto es pro crecimiento con un multiplicador positivo en los sectores de protección social, salud y educación.

Con enfoque de causalidad en el sentido de Granger, Bedir (2016) examina la relación entre gasto en salud y el nivel de ingresos de mercados emergentes en Europa, en Oriente Medio de África y Asia en un período de 1995 a 2013. Sus resultados muestran que el sentido de causalidad es heterogéneo entre países y concluye que, en algunos mercados emergentes, el ingreso es un determinante en el gasto en salud. Asimismo, Dincer y Yüksel (2019) usan los métodos de cointegración y pruebas de causalidad en el sentido de Granger para panel de datos con información de países del $\mathrm{E7}^{2}$, examinan el gasto en salud diferenciando el total, el privado y el público; y, encuentran que existe una relación de largo plazo entre gasto en salud, total y público con $\mathrm{CE}$, y no así, con el gasto privado.

De los principales estudios que se han encontrado para México sobre la relación de salud y CE, está el elaborado por la Comisión Mexicana sobre Macroeconomía y Salud (2006), el cual trata de abordar problemas de salud y sus implicaciones para la economía nacional, se enfoca en el papel que tiene la inversión en salud en la acumulación de capital humano y en las condiciones de pobreza. Dicho estudio encuentra la existencia de un rezago significativo y una gran desigualdad en los indicadores básicos de salud, tales como la tasa de mortalidad infantil y la tasa de mortalidad materna; e identifica la existencia de un grupo de la población en trampa de pobreza.

Por su parte, Mayer-Foulkes (2001) realiza un análisis de causalidad en el sentido de Granger entre CE y salud (medida en expectativa de vida y mortalidad), por grupos de edad y género, con datos de 1950 a 1995. Y encuentra una fuerte causalidad en períodos mayores a los 25 años, de salud hacia el CE; la magnitud del efecto es de dos por ciento. Por otro lado, MayerFoulkes (2008) examina la existencia de trampas de pobreza del capital humano en México, utiliza información de la Encuesta Nacional de Salud para realizar un modelo probit con el que analiza la probabilidad de que jóvenes continúen estudiando más allá de la educación primaria y secundaria; el principal resultado relevante para la actual investigación, es que la salud de los infantes juega un papel importante en la permanencia de los estudios.

En estudios más recientes, se encuentra el de Fuentes-Castillo (2016), quien en su tesis doctoral, examina la relación de largo plazo entre salud y CE en un

2 Países del E7: Brasil, China, India, México, Turquía, Indonesia y Rusia. 
período de 1940 a 2011. Utiliza variables de expectativa de vida, tasa de mortalidad infantil, razón de muerte materna, GPS real y GPS como proporción del PIB. Implementa la técnica de cointegración considerando cambios estructurales. Su principal hallazgo es que existe relación de largo plazo entre CE con la tasa de mortalidad infantil, razón de mortalidad materna y GPS real. Es decir, sus resultados son diferenciados.

En un enfoque más orientado a desarrollo económico, Lorenzo-Valdes y RuizPorras (2017) analizan, con indicadores regionales, la relación dinámica entre producción, natalidad y mortalidad, en un período de 1993 a 2010. Usan pruebas de cointegración y de causalidad en el sentido de Granger, así como estimación de elasticidades mediante DOLS; sus principales resultados son que existe cointegración de la producción con natalidad y mortalidad; que hay correlaciones negativas de la producción y las otras dos variables; que el efecto de la natalidad es mayor que el de la mortalidad; además de que existen efectos de causalidad de la mortalidad hacía la producción, y el efecto es bidireccional entre la natalidad y producción. Estos autores sugieren, según sus hallazgos, que las políticas de salud y de seguridad social contribuyen a promover el crecimiento a largo plazo.

Por su parte, Ruiz-Porras, Aceves-Dávalos y León-Cazares (2017) examinan los fondos de aportación que financian la educación (FAEB), la infraestructura social (FAIS) y la salud (FASSA) mediante técnicas econométricas en panel; entre sus principales hallazgos está que el FAEB incrementa la productividad laboral y el desarrollo económico, mientras que FAIS y FASSA tienen un impacto menor; además encuentran que las transferencias tienen efectos diferenciados en el nivel estatal.

En este contexto, la principal contribución a la literatura del presente trabajo es el análisis de la relación de largo plazo entre CE y GPS desagregado según población objetivo, con y sin SS. Esto permite distinguir características de las instituciones que ofrecen servicios de salud, tales como paquetes de servicios y formas de acceso. Más aún, permite incorporar al análisis características socioeconómicas de la población que recibe servicios de salud de cada tipo de institución.

\section{Metodología}

En referencia a los resultados de investigación identificados en la sección anterior, con objeto de profundizar en el análisis de la relación de gasto en salud y CE se explora dicha relación a partir de un panel de datos con 32 estados de la República Mexicana de 1993 a 2017, con una periodicidad anual. Las variables que se incluyen en dicho panel son Producto Interno Bruto 
(PIB) ${ }^{3}$, gasto público total en salud (GTOTOT), GPS dirigido a personas que no tienen SS (GSINSS), GPS dirigido a personas con SS (GCONSS) ${ }^{4}$ y Población $(\mathrm{POB})^{5}$. Cabe señalar que las variables se transformaron en logaritmo natural por sus beneficios de suavizamiento y de interpretación. Si las variables cumplen las propiedades necesarias para el análisis de largo plazo, se proponen dos modelos para realizar el análisis. El primero incluye el gasto en salud total, y el segundo, incluye el gasto en salud desagregado según su población objetivo, con y sin seguridad social, para poder examinar la relación de largo plazo del gasto, en conjunto y por separado, con el PIB $^{6}$ :

1) Modelo con GPS total:

$\ln \left(P I B_{i t}\right)=\beta_{1} \ln \left(P O B_{i t}\right)+\beta_{2} \ln \left(\right.$ GTOTOT $\left._{i t}\right)+\beta_{3} D+\varepsilon_{i t}$

2) Modelo con GPS desagregado por población objetivo:

$\ln \left(P I B_{i t}\right)=\beta_{1} \ln \left(P O B_{i t}\right)+\beta_{2} \ln \left(\right.$ GSINSS $\left._{i t}\right)+\beta_{3} \ln \left(\right.$ GCONSS $\left._{i t}\right)+$ $\beta_{4} D+\varepsilon_{i t}$

Donde $\ln \left(\right.$ PIB $\left._{i t}\right)$ es el logaritmo natural del PIB, $\ln \left(\right.$ GTOTOT $\left._{i t}\right)$ es el logaritmo natural del GPS total, $\ln \left(\right.$ GSINSS $\left._{i t}\right)$ es el logaritmo natural del GPS en población sin SS, $\ln (G C O N S S)$ es el logaritmo natural del GPS en población con SS, $\ln \left(P O B_{i t}\right)$ es la variable de estado y representa el logaritmo natural de población, $\mathrm{D}$ es una variable que absorbe el efecto de la crisis financiera 2008-2009 y $\varepsilon_{i t}$ es el error. El subíndice i indica la entidad federativa, y $\mathrm{t}$ señala el tiempo.

La metodología está basada en técnicas de series de tiempo adaptadas para panel de datos, ya que este enfoque permite combinar las propiedades de series de tiempo y corte trasversal; a la vez, considera la heterogeneidad de las unidades de medición (entidades federativas) y muestra las dinámicas de cambio, minimiza sesgos de la agregación de datos, reduce la variabilidad y la potencial multicolinealidad de las variables, además, incrementa los grados de libertad y la eficiencia de los estimadores (Baltagi, 2005; Hsiao, 2007, 2014). Con el objeto de identificar características del panel de datos, se aplican técnicas complementarias e independientes. Se inicia haciendo pruebas de raíz unitaria, ya que, si se tienen variables con raíz unitaria o no estacionarias se

3 La variable de PIB fue tomada de Instituto Nacional de Estadística y Geografía (INEGI).

4 Las variables de gasto en salud se obtienen de la Secretaría de Salud.

5 Se obtiene de Consejo Nacional de Población (CONAPO).

6 Por lo regular en la literatura de CE, los modelos empíricos son especificados en términos per cápita, sin embargo, las variables de gasto público en salud per cápita (gasto dividido entre número de beneficiarios) están disponibles a partir del año 2000. Esto implica una reducción considerable en el período de estudio. Aun así, se realizaron las estimaciones en términos per cápita con el objetivo de comparar con los resultados expuestos en el presente trabajo, y fueron similares. 
podría tener análisis erróneos, pues esto podría ocasionar regresiones espurias ${ }^{7}$ o inferencia estadística inválida. Se presentan las pruebas a nivel y en diferencias, esto es necesario para conocer el orden de integración ${ }^{8}$.

Las técnicas para realizar las pruebas de raíz unitaria ${ }^{9}$ implementadas en este estudio son: la propuesta por Levin, Lin y Chu (2002) (LLC), la propuesta por Im, Pesaran y Shin (2003) (IPS), de tipo Fisher-ADF propuesto por Maddala \& Wu (1999) (Fisher-ADF) $)^{10}$, y la propuesta por (Bai \& Carrion-i-Silvestre, 2009) (BCiS). Cabe señalar que las pruebas Fisher-ADF e IPS permiten heterogeneidad entre los individuos, lo cual dadas las características de las entidades federativas podrían ser más apropiadas.

Adicionalmente, la evaluación de la dinámica de los datos a través de la prueba BCiS que permite múltiples quiebres y pendientes diferentes para cada entidad, nos permitiría considerar un alto grado de heterogeneidad ${ }^{11}$. Dado el período de análisis, se requiere analizar que las series puedan contener quiebres estructurales, estos son cambios de parámetros en una serie de tiempo y podrían ocurrir, por ejemplo, como resultado en el régimen de políticas o eventos geopolíticos poco frecuentes pero significativos (Bai \& Carrion-iSilvestre, 2009). Es importante considerar esta posibilidad en el análisis, ya que no hacerlo puede llevar a considerar una variable estacionaria con quiebres estructurales como no estacionaria. Con los resultados de estas pruebas en conjunto se obtiene mayor certeza del orden de integración, resulta de gran importancia identificar esta característica de las variables, ya que la primera condición para que exista una relación no espuria es que sean integradas del mismo orden.

Inclusive, para descartar la existencia de regresiones espurias, se aplican pruebas de cointegración entre las variables de estudio, estas son implementadas para conocer si existe una combinación lineal de dos o más series no estacionarias (Enders, 2015); en otras palabras, identificar si las variables se mueven conjuntamente a lo largo del tiempo y sus diferencias son estables. En este sentido, muestran la presencia de una relación de largo plazo.

7 Las regresiones espurias se caracterizan por tener $\mathrm{R}^{2}$ alto, aunque las variables no estén correlacionadas.

$8 \mathrm{El}$ orden de integración de una serie no estacionaria se refiere al número de veces que se tiene que diferenciar para que sea estacionaria.

9 Se realizan diferentes técnicas de raíz unitaria con el objetivo de obtener mayor robustez. 10 Los supuestos específicos de las pruebas LLC, IPS y Fisher-ADF son descritos en Breitung \& Pesaran (2005).

11 Es preciso mencionar que una limitante de esta técnica en el panel utilizado, es que no se tiene una dimensión en el tiempo muy grande y podría no ser detectado todos lo quiebres estructurales. 
El análisis de cointegración de este estudio sigue la metodología propuesta por Maddala \& Wu (1999) (Fisher-Johansen), es una extensión de la metodología de Johansen (1991): estima los estadísticos de traza y máximo eigenvalor para cada unidad $i(i=1,2,3, . ., \mathrm{N})$. Los valores $\mathrm{p}\left(\pi_{\mathrm{i}}\right)$ de cada estadístico de prueba individual son utilizados para construir los dos estadísticos combinados de las $\mathrm{N}$ unidades. Tanto el estadístico de traza como el de máximo eigenvalor siguen la misma distribución, $-2 \sum_{i=1}^{N} \log \left(\pi_{i}\right) \rightarrow \chi_{2 N}^{2}$, y son utilizados para evaluar la hipótesis nula de no cointegración, así como el número de relaciones de cointegración. De acuerdo con la heterogeneidad de las entidades federativas, esta metodología se considera adecuada ${ }^{12}$.

Se han desarrollado diferentes tipos de pruebas de cointegración basada en los residuales para paneles de datos, uno de los más conocidos es el de Pedroni $(1999,2004)$, este se basa en los residuales y desarrolla sietes estadísticos de pruebas. La estimación se realiza utilizando Mínimos Cuadrados Ordinarios Completamente Modificados Agrupados (PFMOLS, por sus siglas en inglés), aunque considera heterogeneidad permitiendo que el intercepto y la pendiente varíen entre las unidades, si la relación de largo plazo es heterogénea entre las unidades se producirán pruebas inconsistentes (Pedroni, 2019). En este sentido, se considera que esta técnica no es adecuada para el contexto de las entidades federativas.

Sin embargo, las técnicas de cointegración antes mencionadas no consideran cambios estructurales, y esto puede llevar a inferencias engañosas en las pruebas. De hecho, las pruebas que distinguen entre procesos espurios o cointegrados tienden a favorecer los modelos espurios cuando el proceso está cointegrado dentro del régimen de ruptura (Westerlund \& Edgerton, 2008). Ante la posibilidad de esta situación, de manera complementaria, se implementa el método de cointegración propuesto por Westerlund y Edgerton (2008) (WE) que considera quiebres estructurales no conocidos. Individualmente, se estima punto de quiebre para cada miembro del panel, en diferentes fechas ${ }^{13}$. Cabe señalar que esta técnica permite heterogeneidad y autocorrelación en los errores, así como dependencia trasversal. De esta manera, se determina si existe una relación de largo plazo entre las variables de estudio, incluso ante la existencia de dichos quiebres.

Es importante notar que mientras se estiman elasticidades del PIB, respecto de los tipos de gasto en salud, realizar estas estimaciones por Mínimos Cuadrados Ordinarios con variables no estacionarias podría llevar a tener sesgos y su distribución depende de parámetros que no permiten hacer inferencia. En

12 Existen estudios hechos con paneles de datos con el PIB de los Estados mexicanos, que utilizan esta metodología y han encontrado resultados robustos (véase Lorenzo-Valdes \& Ruiz-Porras (2017) y Rodríguez-Benavides \& Mendoza-González (2015) .

13 Sigue la estrategia de Bai \& Perron (1998) para localizar los puntos de quiebre. 
particular, Pedroni (2001) expone que estos parámetros son regresores que no son parte del verdadero proceso generador de datos, pero que podrían causar endogeneidad y correlación en el modelo. Para abordar este problema, se han desarrollado diferentes enfoques para panel, el de Mínimos Cuadrados Ordinarios Dinámicos (DOLS, por sus siglas en inglés) y Mínimos Cuadrados Ordinarios Completamente Modificados (FMOLS, por sus siglas en inglés). El primero, estima de manera paramétrica incluyendo adelantos y rezagos de las diferencias de los regresores resolviendo el problema de endogeneidad y autocorrelación serial. El segundo, también resuelve este problema, pero mediante un tratamiento no paramétrico.

Incidentalmente, dentro de los enfoques de DOLS y FMOLS en panel, existe variación en la forma de estimar, agrupados y medias de grupo. Los agrupados pueden suponer completa homogeneidad entre las unidades y contemplar heterogeneidad dejando que las constantes y la pendiente sean diferentes. De acuerdo con Pedroni $(2001,2019)$, la técnica de datos agrupados supone que la relación de cointegración es la misma para todas la unidades. Para resolver esta situación, este autor propone el enfoque de medias de grupo (MG). Básicamente, consiste en generar el estimador para cada una de las unidades y hacer un promedio, proporcionando heterogeneidad, no solo en la constante y la pendiente, sino en la relación de cointegración.

Por lo anterior, MGFMOLS se consideran adecuados para el objetivo de este trabajo, no solo por la heterogeneidad que se podría contemplar entre las entidades federativas, sino también porque un tema discutido en la literatura es la endogeneidad originada por la causalidad de reversa del PIB hacia el gasto público en salud, pues si esta variable crece, también los ingresos públicos y por lo tanto el gasto público. Pedroni (2019) comenta que, la estrategia principal es ajustar el sesgo que surge de la retroalimentación dinámica, debido a la endogeneidad de los regresores mediante el uso de la dinámica de los mismos como un instrumento interno, resolviendo así la endogeneidad completa. Una ventaja de los MGFMOLS es que sufre de poca distorsión en muestras pequeñas (Pedroni, 2002). En este contexto, se implementa MGFMOLS para realizar las estimaciones de los coeficientes de largo plazo, que, al estar transformadas a logaritmos, se consideran las elasticidades de largo plazo del PIB respecto al gasto público en salud, además de proporcionar estimadores individuales de las entidades federativas.

En los resultados se presentan las elasticidades de largo plazo para el panel completo, y las correspondientes a cada entidad federativa. En un esfuerzo por caracterizar dichas elasticidades en los estados, se incluyen variables las cuales no pertenecen al modelo, pero sí a características de las entidades. Estas características que se consideran pueden ayudar a explorar una posible asociación con la productividad del GPS, que son las siguientes: nivel de 
desarrollo (ND) ${ }^{14}$, grado de marginación $(\mathrm{GM})^{15}$, coeficiente de Gini $(\mathrm{CG})$ e índice de desarrollo humano (IDH) ${ }^{16}$. Se agruparán las entidades federativas por nivel de desarrollo: bajo, medio y alto.

Como se menciona anteriormente, un tema debatido es el sentido de causalidad de las variables de estudios, para esto se toma la noción de Granger (1969). Si las variables son estacionarias, la prueba de causalidad en el sentido de Granger puede hacerse mediante un Vector Autorregresivo (VAR), sin embargo, si las variables son no estacionarias y cointegradas, y se hace en primeras diferencias estaría mal especificado, ya que al aplicar diferencias se remueve la información de largo plazo, la forma de abordar este problema es mediante un Vector de Corrección de Errores en Panel (PVEC, por sus siglas en inglés) (Engle \& Granger, 1987). Cabe mencionar que esta técnica puede identificar la relación de largo y corto plazo, además permite introducir efectos fijos. Así, esta técnica es utilizada en el presente trabajo para determinar el efecto causal, de corto y largo plazo.

La causalidad de largo plazo puede ser obtenida en el modelo VEC mediante el Término de Corrección de Error (ECT, por sus siglas en inglés), si es negativo y estadísticamente significativo, entonces hay un efecto de causalidad de las variables independientes hacia la variable dependiente. Para la causalidad en el corto plazo, se realiza la prueba de Wald para investigar si los coeficientes de corto plazo son estadísticamente significativos.

\section{Resultados ${ }^{17}$}

En cuanto a la realización de las pruebas de raíz unitaria LLC, IPS, FisherADF y BCiS, la hipótesis nula es que existe raíz unitaria. El cuadro 1 muestra los resultados que permiten evaluar si las variables PIB, GCONSS, GSINSS y POB son no estacionarias a nivel ${ }^{18}$. En general, todas las pruebas indican la ausencia de raíz unitaria en primeras diferencias, es decir, son integradas de primer orden, I(1); por lo tanto, son elegibles para realizar el análisis de cointegración.

Las pruebas de cointegración que se reportan en el cuadro 2, muestran si las variables de estudio, a pesar de ser no estacionarias, tienen movimientos en

14 Véase en Padilla-Hermida y Garrido-Noguera (2010).

15 El coeficiente de Gini y el Grado de marginación son publicados por Consejo Nacional de Población (CONAPO).

16 IDH publicado por (De la Torre García et al., 2015).

17 Los cálculos y resultados a detalle están disponibles con el autor por correspondencia.

18 Los resultados de las pruebas de raíz unitaria no son concluyentes para GTOTOT. Por esta razón, el modelo de la ecuación 1 (que sería utilizado para contrastar el modelo de la ecuación 2) no se estima. 
común. En el cuadro 2a, se muestra la prueba Fisher-Johansen, se evalúa la hipótesis nula de no cointegración y el número de relaciones de cointegración existentes entre las variables en estudio, dicha evaluación se hace con base en los estadísticos combinados de traza y eigenvalor. Los resultados sugieren que hay dos o tres relaciones de cointegración. Por lo que este resultado sugiere que PIB, GSINSS, GCONSS y POB están cointegradas.

\section{Cuadro 1}

Pruebas de raíz unitaria

\begin{tabular}{|c|c|c|c|c|c|}
\hline \multirow{2}{*}{ Variable } & \multicolumn{2}{|c|}{ A nivel } & \multicolumn{2}{|c|}{ Primera diferencia } & \multirow{2}{*}{ Orden } \\
\hline & Estadístico & Probabilidad & Estadístico & Probabilidad & \\
\hline \multicolumn{6}{|c|}{ 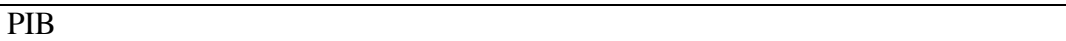 } \\
\hline LLC & -0.4533 & 0.3252 & -24.7087 & 0 & 1 \\
\hline IPS & 6.4583 & 1 & -25.8564 & 0 & 1 \\
\hline Fisher-ADF & 8.0347 & 1 & 621.5558 & 0 & 1 \\
\hline BCiS (est-Z) & 0.383 & 0.371 & -4.2024907 & 0 & \\
\hline BCiS (est-Pm) & -0.459 & 0.359 & 10.42 & 0 & \\
\hline \multicolumn{6}{|l|}{ GTOTOT } \\
\hline LLC & -3.1347 & 0.0009 & -19.4932 & 0 & 0 \\
\hline IPS & 3.6284 & 0.9999 & -20.4369 & 0 & 1 \\
\hline Fisher-ADF & 19.9105 & 1 & 620.8552 & 0 & 1 \\
\hline BCiS (est-Z) & -2.4200 & 0.0213 & -4.40 & 0 & \\
\hline BCiS (est-Pm) & 4.410 & 0.000 & 16.35 & 0 & \\
\hline \multicolumn{6}{|l|}{ GSINSS } \\
\hline LLC & 0.7489 & 0.773 & -20.4099 & 0 & 1 \\
\hline IPS & 7.1911 & 1 & -21.3515 & 0 & 1 \\
\hline Fisher-ADF & 58.5534 & 0.6687 & 602.1615 & 0 & 1 \\
\hline BCiS (est-Z) & 0.1622 & 0.3937 & -4.8086253 & 0 & 1 \\
\hline BCiS (est-Pm) & 0.148 & 0.395 & 17.78 & 0 & \\
\hline \multicolumn{6}{|l|}{ GCONSS } \\
\hline LLC & -0.4918 & 0.3114 & -21.545 & 0 & 1 \\
\hline IPS & 5.5643 & 1 & -22.6588 & 0 & 1 \\
\hline Fisher-ADF & 17.1255 & 1 & 690.7351 & 0 & 1 \\
\hline BCiS (est-Z) & -1.5767 & 0.1151 & -3.8024313 & 0 & 1 \\
\hline BCiS (est-Pm) & 0.890 & 0.268 & 11.72 & 0 & \\
\hline \multicolumn{6}{|l|}{ POB } \\
\hline LLC & -4.2663 & 0 & -7.1195 & 0 & 0 \\
\hline IPS & 3.7157 & 0.9999 & -5.2034 & 0 & 1 \\
\hline Fisher-ADF & 45.7199 & 0.9592 & 696.1035 & 0 & 1 \\
\hline BCiS (est-Z) & 0.633 & 0.32651381 & 10.601 & 0 & \\
\hline BCiS (est-Pm) & -0.360 & 0.374 & -3.67 & 0 & \\
\hline
\end{tabular}

Nota: El número de rezagos fue determinado mediante el Criterio de Información de Schwarz.

De manera complementaria, para identificar si las variables están cointegradas aun en presencia de quiebres estructurales, se hace la prueba de Westerlund \& Edgerton (2008). En el cuadro 2b se muestran los resultados considerando que 
no tenga quiebre, con quiebre en el nivel o quiebre en el régimen. El estadístico de $Z \tau$ sugiere cointegración sin quiebres y con quiebres en el nivel; lo mismo sugieren ambos estadísticos, $\mathrm{Z} \tau$ y $\mathrm{Z} \varphi$, con quiebres en el régimen. Con los resultados de las dos pruebas de cointegración, se encuentra evidencia a favor de la existencia de relación de largo plazo entre las variables en estudio.

\section{Cuadro 2}

Pruebas de cointegración para panel

\begin{tabular}{|c|c|c|c|c|}
\hline \multicolumn{5}{|c|}{ a) Prueba de cointegración (Fisher-Johansen) } \\
\hline & \multicolumn{2}{|c|}{ Pruebas de traza } & \multicolumn{2}{|c|}{ Pruebas de eigenvalor } \\
\hline & Estadístico & Probabilidad* & Estadístico & Probabilidad* \\
\hline Ninguna & 817.7 & 0 & 817.7 & 0 \\
\hline A lo más 1 & 334.5 & 0 & 334.5 & 0 \\
\hline A lo más 2 & 142.8 & 0 & 142.8 & 0 \\
\hline A lo más 3 & 70.78 & 0.2619 & 70.78 & 0.2619 \\
\hline \multicolumn{5}{|c|}{ b) Prueba de cointegración con quiebre estructural (WE) } \\
\hline & $\mathrm{Z} \tau$ & Probabilidad & $\mathrm{Z} \phi$ & Probabilidad \\
\hline Sin quiebre & -3.906 & 0.000 & -1.048 & 0.147 \\
\hline $\begin{array}{l}\text { Con quiebres a } \\
\text { nivel } \\
\text { Con quiebre en }\end{array}$ & -1.31 & 0.095 & 0.347 & 0.636 \\
\hline regimen & -4.12 & 0.000 & -2.97 & 0.001 \\
\hline
\end{tabular}

Nota: *La probabilidad se calcula usando una distribución Chi-cuadrada.

Una vez que se tiene evidencia de que las variables tienen movimientos comunes y relación de largo plazo, se procede a la estimación de elasticidades de largo plazo del PIB, con respecto a los gastos y la población. En el cuadro 3 , se muestran dichas estimaciones, los valores de la última fila corresponden a los coeficientes para todo el panel, estos son el promedio de los coeficientes individuales de cada estado. Indican que, cuando el gasto público en salud destinado a la población CONSS incrementa en uno por ciento, el PIB incrementa en 0.50 por ciento; mientras que al incremento del uno por ciento del GSINSS, el PIB incrementa el 0.16 por ciento ${ }^{19}$. Los tres coeficientes son estadísticamente significativos. A nivel estatal, mientras que Campeche reporta la mayor productividad de ambos GPS (GSINSS de 0.83 y GCONSS 2.92); Coahuila, Colima y Querétaro muestran elasticidad negativa en el GSINSS de $-0.04,-0.03$ y -0.02 , respectivamente; de igual manera. Por otro lado, Chiapas y Sonora muestran elasticidad negativa ${ }^{20}$ en el GCONSS de 0.17 y -0.08 , respectivamente.

19 Puede ser interesante notar que si se suman los coeficientes de GCONSS y GSINSS, el resultado conjunto muestra un efecto del GPS sobre el PIB que es cercano al estimado por el de Wang (2011), para México (0.86).

20 Tabasco y Baja California también presentan elasticidad negativa en GCONSS, pero no son estadísticamente significativas. 
Visto desde la perspectiva del capital humano, si el gasto en salud propicia la acumulación de capital humano, y así, la productividad laboral se traduce en crecimiento económico, es de esperarse que los rendimientos de cada tipo de gasto en salud sean disimiles debido a las considerables brechas entre los derechos de atención. En el trabajo de Durán-Arenas (2012), se presentan tres tipos de desigualdad: 1) en el acceso a servicio de salud, que se da en el nivel regional, pues en zonas menos desarrolladas cuentan con menos infraestructura y personal médico por habitante, que en ciudades más importantes del país; 2) en paquetes de beneficios, por ejemplo, el Seguro Popular solo cubría el 12.8 $\%$ de los diagnósticos que cubren el IMSS y el ISSSTE; y 3 ) en calidad de la atención médica, ya que existen estudios donde se evalúa la calidad en tratamientos específicos por institución que dejan a la luz esta desigualdad. Como ya se había mencionado, en un estudio más reciente hecho por la OCDE (2016), se exponen algunas diferencias en el acceso y otras características.

Por otro lado, la diferencia entre las elasticidades del GCONSS y GSINSS sugerimos podría estar relacionada con la distorsión que ocasiona en el mercado laboral. De acuerdo con Levy (2008), la combinación de seguridad social y protección social induce a los trabajadores y empresas a trabajar e invertir en actividades informales que impiden el crecimiento de la productividad, genera incentivos para trabajadores y empresas a violar las leyes orientadas a proteger a los trabajadores, y falla en reducir efectivamente la desigualdad en el ingreso. Por un lado, se grava al empleo formal y por otro, se subsidia al informal (Busso et al., 2012; Levy, 2008). Cabe señalar que el ingreso de las instituciones de seguridad social proviene de subsidios del gobierno, y de aportaciones de la empresa y de los trabajadores; mientras que el ingreso de las instituciones desvinculadas del trabajo, viene de subsidio y una aportación de quien recibe el servicio.

De acuerdo con lo anteriormente expuesto, otra explicación sugerida que podría tener el hecho de que la elasticidad de GSINSS sea menor a la de GCONSS, es que el primero funciona como un incentivo para los trabajadores a operar en el sector informal, cuando un individuo tiene que elegir entre un empleo formal e informal, no solo pondera el salario, sino los beneficios entre ambos sectores; si es adverso al riesgo elegirá el sector formal, por los beneficios que ofrece. Sin embargo, al existir servicios de salud que son subsidiados, se podría evadir contribuir a la seguridad social y elegir el sector informal mientras recibe servicios de salud de instituciones sin seguridad social. Por ejemplo, el estudio de Aterido et al. (2011), encuentra que el programa del Seguro Popular disminuye el flujo de trabajadores al sector formal, en un 20 por ciento.

En lo que sigue, las elasticidades de largo plazo en el nivel nacional serán utilizadas como elementos a asociar con los niveles de desarrollo socio- 
económico de las entidades federativas. Para llevar a cabo dicho análisis hemos identificado variables con características socioeconómicas de las entidades federativas y que no fueron incluidas en la estimación del modelo por dos razones; en primer lugar, la información no está disponible para cada año; y en segundo, la técnica utilizada para estimar las elasticidades, básicamente se genera del estimador individual y se promedian para obtener el estimador del panel completo, de modo que una variable dicotómica o categórica para clasificar los estados, tendría poca utilidad. Sin embargo, con estas variables se pretende captar información de los estados que podría asociarse con la magnitud de las elasticidades encontradas previamente en el estudio.

En particular, en el cuadro 3, se agrupan las entidades de acuerdo con la clasificación de desarrollo financiero propuesto por Padilla-Hermida \& Garrido-Noguera (2010), que se considera pertinente ya que incluye variables socioeconómicas para la construcción de los grupos de los estados. Centrando primero el análisis en las entidades catalogadas de bajo desarrollo, llama la atención que, en la mayoría de estos estados, las elasticidades identificadas previamente en el estudio son mayores a la media nacional, dicho en otras palabras, son aquellos donde el gasto en salud es más productivo o tiene mayores rendimientos de manera conjunta.

Cabe señalar que se muestran algunas excepciones como Tabasco, Quintana Roo y Baja California Sur, donde el GCONSS no es significativo y si lo es el GSINSS, en estos estados el coeficiente de Gini es mayor o muy cercano a la media nacional. En el caso de Colima, ambos gastos no son significativos, sin embargo, es de los estados con IM bajo, con menor índice de Gini y un IDH muy alto. Chiapas tiene una elasticidad negativa respecto a GCONSS, nótese que es de los estados con Gini e IM muy alto, así como IDH bajo.

Es de notar que el GSINSS, en su naturaleza, tiene como objetivo proveer de servicios a la población más desprotegida, que no tiene afiliación a la seguridad social y que, por lo regular, no puede o le es muy difícil recurrir al sector privado de salud. Teniendo esto en mente, la productividad de este gasto es de algún modo mayor en el grupo de estados de bajo desarrollo, que, según el coeficiente de Gini, en promedio es donde hay mayor desigualdad. En este contexto, este gasto podría funcionar como un mecanismo redistributivo, que con mejoras en los paquetes y calidad de servicios podría contribuir en gran medida al desarrollo de estas entidades. 


\section{Cuadro 3}

Elasticidades de largo plazo (Variable independiente: LPIB)

\begin{tabular}{|c|c|c|c|c|c|c|c|}
\hline \multirow[b]{2}{*}{ Estado } & \multicolumn{3}{|c|}{$\begin{array}{l}\text { Variables del Modelo } \\
\text { LGCONS }\end{array}$} & \multicolumn{4}{|c|}{$\begin{array}{c}\text { Caractéristicas de la entidades } \\
\text { Gini20 }\end{array}$} \\
\hline & LGSINSS & $\mathbf{S}$ & LPOB & CDE 1 & IM $^{2}$ & $12^{3}$ & IDH $^{4}$ \\
\hline Tabasco & $0.44 * * *$ & -0.47 & $3.74 * * *$ & Bajo & Medio & 0.516 & Medio \\
\hline $\begin{array}{l}\text { Quintana Roo } \\
\text { Baja }\end{array}$ & $0.10 * * *$ & 0.06 & $1.09 * * *$ & Bajo & Medio & 0.477 & $\begin{array}{l}\text { Alto } \\
\text { Muy }\end{array}$ \\
\hline $\begin{array}{l}\text { California Sur } \\
\text { Michoacán de }\end{array}$ & $0.30 * * *$ & 0.18 & $1.24 * * *$ & Bajo & Bajo & 0.493 & Alto \\
\hline Oсатро & $0.28 * * *$ & $0.28 * * *$ & $1.14 * * *$ & Bajo & Alto & 0.472 & Bajo \\
\hline Morelos & $0.24 * * *$ & $0.47 * * *$ & $-0.3 * *$ & Bajo & Medio & 0.433 & Alto \\
\hline Yucatán & $0.19 * * *$ & $0.46^{* * *}$ & $1.14 * * *$ & Bajo & Alto & 0.461 & Medio \\
\hline Durango & $0.12 * * *$ & $0.53^{* * * *}$ & $1.61 * * *$ & Bajo & $\begin{array}{l}\text { Medio } \\
\text { Muy }\end{array}$ & 0.499 & Medio \\
\hline Guerrero & $0.20 * * *$ & $0.61 * * *$ & $-0.41 * * *$ & Bajo & alto & 0.533 & Bajo \\
\hline Tlaxcala & $0.15 * * *$ & $0.71 * * *$ & $0.55^{* *}$ & Bajo & Medio & 0.42 & $\begin{array}{c}\text { Medio } \\
\text { Muy }\end{array}$ \\
\hline Colima & -0.03 & 0.06 & $2.59 * * *$ & Bajo & Bajo & 0.445 & Alto \\
\hline Hidalgo & $0.10 * * *$ & $0.75^{* * *}$ & $1.24 * * *$ & Bajo & Alto & 0.48 & Medio \\
\hline Zacatecas & 0.07 & $0.81 * * *$ & $2.64 * * *$ & Bajo & Medio & 0.526 & Bajo \\
\hline Nayarit & $0.32 * * *$ & $0.9 * * *$ & $0.33 *$ & Bajo & $\begin{array}{l}\text { Medio } \\
\text { Muy }\end{array}$ & 0.498 & Medio \\
\hline Oaxaca & $0.21 * * *$ & $0.89 * * *$ & -0.16 & Bajo & $\begin{array}{l}\text { alto } \\
\text { Muy }\end{array}$ & 0.511 & Bajo \\
\hline Chiapas & $0.1 * * *$ & $-0.17 * * *$ & $3.12 * * *$ & Bajo & alto & 0.535 & Bajo \\
\hline Campeche & $0.83 * * *$ & $2.92 * * *$ & $-4.22 * * *$ & Bajo & Alto & 0.533 & $\begin{array}{l}\text { Alto } \\
\text { Muy }\end{array}$ \\
\hline Sonora & $0.11 * * *$ & $-0.08 * *$ & $3.29 * * *$ & Medio & Bajo & 0.477 & Alto \\
\hline $\begin{array}{l}\text { Baja } \\
\text { California }\end{array}$ & $0.11 * * *$ & -0.05 & $1.57 * * *$ & Medio & $\begin{array}{l}\text { Muy } \\
\text { bajo }\end{array}$ & 0.465 & $\begin{array}{l}\text { Muy } \\
\text { Alto }\end{array}$ \\
\hline Aguascalientes & $0.05 * * *$ & 0.02 & $2.08 * * *$ & Medio & Bajo & 0.479 & $\begin{array}{l}\text { Alto } \\
\text { Muy }\end{array}$ \\
\hline $\begin{array}{l}\text { Querétaro } \\
\text { San Luis }\end{array}$ & -0.02 & $0.48 * * *$ & $2 * * *$ & Medio & Bajo & 0.503 & Alto \\
\hline Potosí & 0.05 & $0.58 * * *$ & $2.93 * * *$ & Medio & Alto & 0.492 & Medio \\
\hline Guanajuato & $0.06 * * *$ & $0.56 * * *$ & $2.01 * * *$ & Medio & Medio & 0.463 & Bajo \\
\hline Tamaulipas & 0.09 & $0.67 * * *$ & $1.18 * *$ & Medio & Bajo & 0.466 & Alto \\
\hline Veracruz & $0.09 *$ & $0.68 * * *$ & $2.38 * * *$ & Medio & Alto & 0.493 & Bajo \\
\hline Sinaloa & $0.13 * * *$ & $1.05^{* * *}$ & $1.24 * * *$ & Medio & Medio & 0.466 & Alto \\
\hline $\begin{array}{l}\text { Coahuila de } \\
\text { Zaragoza }\end{array}$ & -0.04 & $1.09 * * *$ & $1.35 * * *$ & Alto & $\begin{array}{l}\text { Muy } \\
\text { bajo }\end{array}$ & 0.464 & $\begin{array}{l}\text { Muy } \\
\text { Alto }\end{array}$ \\
\hline Chihuahua & $0.08^{* * *}$ & $0.77 * * *$ & $1.24 * * *$ & Alto & Bajo & 0.5 & Medio \\
\hline Distrito & & & & & Muy & & Muy \\
\hline Federal & $0.06 * * *$ & $0.09 * * *$ & $6.48 * * *$ & Alto & bajo & 0.457 & Alto \\
\hline México & $0.09 * * *$ & $0.2 * * *$ & $0.99 * * *$ & Alto & Bajo & 0.47 & Alto \\
\hline Puebla & $0.13 * * *$ & $0.23 * * *$ & $1.64 * * *$ & Alto & $\begin{array}{l}\text { Alto } \\
\text { Muy }\end{array}$ & 0.485 & $\begin{array}{l}\text { Bajo } \\
\text { Muy }\end{array}$ \\
\hline Nuevo León & $0.33 * * *$ & $0.33 * * *$ & $0.77 * * *$ & Alto & bajo & 0.485 & Alto \\
\hline Jalisco & $0.09 * * *$ & $0.5 * * *$ & $1.83 * * *$ & Alto & Bajo & 0.473 & Alto \\
\hline Nacional & $0.16 * * *$ & $0.50 * * *$ & $1.51 * * *$ & & & 0.49 & \\
\hline
\end{tabular}

Los "***", "**" y "*" denotan significancia al 1\%, 5\% y $10 \%$ respectivamente. (1) Clasificación de acuerdo a su desarrollo económico: bajo, medio y alto. (2) Grado de Marginación. (3) Coeficiente de Gini, indica el grado de desigualdad respecto al ingreso, a más se acerque a 1 mayor es la desigualdad. (4) Índice de Desarrollo Humano. 
Por otro lado, las entidades clasificadas con desarrollo medio tienen elasticidad del GSINSS menor a la media nacional y en algunos casos no son estadísticamente significativas, como son los casos de Tamaulipas, Querétaro, y San Luis Potosí. El GCONSS tiene elasticidades por encima de la media nacional, siendo este el más productivo en este grupo de estados. En los casos de Aguascalientes y Baja California, no es estadísticamente significativo. Como se puede apreciar, la relación de las elasticidades con características socioeconómicas no es tan clara como en los estados de desarrollo bajo, sin embargo, se puede considerar que las entidades de desarrollo medio tienen mayor relevancia el GCONSS, en cuanto a la productividad de los gastos en salud.

En el caso de los estados con desarrollo alto, la mayoría tiene elasticidades menores a la media, lo que sugiere una menor productividad del gasto y/o rendimientos decrecientes del nivel de GS. Cabe señalar que, en este grupo de estados, en comparación con los otros grupos, tienen en promedio un coeficiente de Gini más bajo, todos los estados tienen un IM bajo o muy bajo y, en su mayoría IDH alto o muy alto. El caso particular de Coahuila, la elasticidad del GSINSS no es estadísticamente significativa, pero el PIB es más sensible a cambios del GCONSS, pues tiene una elasticidad mayor a 1; es de mencionar que tiene un IM muy bajo, IDH muy alto y el coeficiente de Gini más bajo de este grupo, después del Distrito Federal.

Adicionalmente, se pretende abonar a la literatura sobre el sentido de causalidad entre gasto público en salud y crecimiento económico, es decir, mayor gasto en salud conduce a un mayor crecimiento o viceversa. Para esto, se realiza un análisis de causalidad en el sentido de Granger, a partir de un modelo VEC, para su estimación se selecciona el número de rezagos óptimos con el criterio de información de Akaike y el de Schwarz. Posterior a la estimación del modelo VEC, se desarrolla la prueba de correlación serial de Breusch-Godfrey $(\mathrm{LM})^{21}$, para confirmar la validez de sus estimaciones (Wooldridge, 2010). Los resultados de las pruebas de causalidad son mostrados en el cuadro 4.

El cuadro 4 muestra que el coeficiente de largo plazo es negativo y estadísticamente significativo para el caso de PIB y el GSINSS como variable dependiente. Esto sugiere que 1) GSINSS, GCONSS y POB causan en el sentido de Granger, al PIB en el largo plazo (GSINSS, GCONSS y POB $\rightarrow$ PIB), y 2)PIB, GCONSS y POB causan en el sentido de Granger, al GSINSS en el largo plazo (PIB, GCONSS y POB $\rightarrow$ GSINSS). Por lo contrario, no hay

21 La hipótesis nula es no correlación serial, la cual no fue rechazada con un estadístico LM de 17.46 (prob. 0.35). 
evidencia de relación de largo plazo cuando POB y GCONSS son variables dependientes.

\section{Cuadro 4}

Pruebas de causalidad en el sentido de Granger

\begin{tabular}{|c|c|c|c|c|c|}
\hline \multirow[b]{3}{*}{ Variable dependiente } & \multicolumn{5}{|c|}{ Dirección de causalidad/ variables explicativas } \\
\hline & \multicolumn{4}{|c|}{ Estadísticos $\mathrm{X}^{2} \quad$ (Valor P) } & $\begin{array}{l}\text { Coeficiente } \\
\text { de largo } \\
\text { plazo } \\
\text { (Estadístico } \\
\text { t) }\end{array}$ \\
\hline & LPIB & LPOB & LGCONSS & LGSINSS & \\
\hline LPIB & & $\begin{array}{c}4.49 \\
(0.81)\end{array}$ & $\begin{array}{r}62.62 \\
(0.00)\end{array}$ & $\begin{array}{l}27.97 \\
(0.00)\end{array}$ & $\begin{array}{c}-0.013 * * \\
(-2.10)\end{array}$ \\
\hline LPOB & $\begin{array}{c}7.21 \\
(0.51)\end{array}$ & & $\begin{array}{l}20.01 \\
(0.01)\end{array}$ & $\begin{array}{c}8.69 \\
(0.37)\end{array}$ & $\begin{array}{l}0.0007 \\
(1.65)\end{array}$ \\
\hline LGCONSS & $\begin{array}{l}20.17 \\
(0.01)\end{array}$ & $\begin{array}{l}14.24 \\
(0.08)\end{array}$ & & $\begin{array}{l}26.29 \\
(0.00)\end{array}$ & $\begin{array}{c}0.0008 \\
(1.20)\end{array}$ \\
\hline LGSINSS & $\begin{array}{l}41.53 \\
(0.00)\end{array}$ & $\begin{array}{c}9.37 \\
(0.31)\end{array}$ & $\begin{array}{c}7.75 \\
(0.46)\end{array}$ & & $\begin{array}{c}-0.034 * * * \\
(-5.17)\end{array}$ \\
\hline
\end{tabular}

Corto plazo. Se presenta el estadístico X2 de la prueba de Wald y su valor $\mathrm{p}$ entre paréntesis (). Largo plazo: Se reporta el coeficiente del ECT y su estadístico t en paréntesis. Los "***", "**" y $" * "$ denotan significancia de $1 \%, 5 \%$ y $10 \%$ respectivamente.

La causalidad bidireccional en el sentido de Granger, de corto y largo plazo se refuerza mutuamente en el caso de PIB y GSINSS. En la relación de causalidad entre PIB y GCONSS es bidireccional solo en el corto plazo. Estos resultados coinciden con estudios como el de Rivera \& Currais $(1999,2003)$ y el de Rahman (2011).

Paradójicamente, el gasto que sostiene bidireccionalidad con el PIB es el GSINSS, esto es así, no solo por las mejoras en salud hacía los beneficiarios de los servicios que presta, sino también debido a la forma en que es financiado, ya que este gasto proviene de recursos federales y estatales, y con un aporte del beneficiario. En cambio, el GCONSS procede de recursos federales, empresa y trabajador. En este sentido, los resultados apoyan la propuesta de Levy (2008), sobre redefinir la seguridad social, tanto en su alcance como en la forma que es financiado, y otorgar derechos sociales universales financiados por impuestos al consumo. 


\section{Conclusión}

Mientras que el objetivo de este trabajo fue analizar la relación de largo plazo entre CE y GPS, la principal contribución a la literatura es que al desagregar el GPS, según población objetivo, se distinguen las características de las instituciones que les ofrecen servicios de salud, tanto en sus paquetes de servicios como en la forma de acceso (contributivo o subsidiado), así como por las características laborales formales de la población que recibe servicios de salud, obtenemos resultados más específicos que condicionan la relación GPS y CE.

Los resultados se obtuvieron mediante el empleo de técnicas de series de tiempo, adecuadas para panel de datos, específicamente, pruebas de raíz unitaria y de cointegración, así como estimación de elasticidades y pruebas de causalidad. El estudio, en particular, se sustenta en un panel de datos con indicadores anuales de entidades federativas en el período de 1993 a 2017.

El primer resultado que se obtiene es la evidencia de movimientos comunes y equilibrio de largo plazo entre el PIB y ambos GPS. Otros resultados muestran que la estimación de las elasticidades en el nivel nacional indica mayor productividad del gasto dirigido a la población con seguridad social, en comparación con el gasto destinado a la población sin seguridad social, lo que puede estar asociado a las brechas entre los derechos de atención que ofrece cada tipo de institución. Este resultado apoya el argumento del estudio elaborado por la OCDE (2016), en el que se expone que México corre el riesgo de afianzar la desigualdad socioeconómica con un sistema de salud con marcadas diferencias en calidad y acceso.

Adicionalmente, los resultados sugieren a través del análisis de las elasticidades a nivel estatal, que el efecto del GPS es disímil entre las entidades federativas, se observa que esas diferencias pudieran estar asociadas al nivel de desarrollo socioeconómico de los estados. Así, por ejemplo, los dos tipos de GPS son más productivos en estados de bajo desarrollo, mientras que en los de desarrollo medio, el GPS dirigido a la SS cobra más relevancia en cuanto a la productividad; en los estados de desarrollo alto, ambos GPS son relevantes, pero con una productividad menor que la de los otros dos grupos.

Si se considera el estatus socioeconómico de la población a quien va dirigido el GSINSS, se trata de personas que participan en el mercado informal y no tienen acceso, o les es muy difícil recurrir a servicios de salud privados; en este sentido, promover este gasto, especialmente en estados de bajo desarrollo, podría explorarse como instrumento de política para mitigar las trampas intergeneracionales de las que trata (Mayer-Foulkes, 2008); además de tener un impacto positivo en el crecimiento. 
La evidencia presentada muestra que el análisis en el nivel regional y diferenciando el GPS por población objetivo, proporciona más información que puede contribuir a la generación de políticas públicas específicas para cada entidad federativa. Por ejemplo, en estados donde existe mayor desigualdad y el efecto del GSINSS es mayor, es conveniente promover este gasto ya que contribuiría a la disminución de la desigualdad e incentivaría el CE. Sin embargo, este estudio no es conclusivo, es necesario obtener más información con respecto a las problemáticas de salud específicas de cada entidad, para generar programas más eficientes.

Una de las limitaciones de este estudio, es que al utilizar indicadores agregados no se captan características particulares de individuos, por lo que es conveniente para futuras investigaciones, realizar el análisis utilizando microdatos. Otra futura línea de investigación podría ser examinar los mecanismos adyacentes en los que el GPS interactúa con el CE; por ejemplo, examinar los GPS y su interacción con el mercado laboral, así como indicadores de pobreza.

\section{Referencias}

[1] Abdullah, S. M., Siddiqua, S., \& Huque, R. (2017). Is health care a necessary or luxury product for Asian countries? An answer using panel approach. Health Economics Review, 7(4). https://doi.org/10.1186/s13561-017-0144-8

[2] Acemoglu, D., \& Johnson, S. (2006). Disease and Development: The Effect of Life Expectancy on Economic Growth (N. ${ }^{\circ}$ 12269; NBER Working Paper). http://www.nber.org/papers/w12269

[3] Amiri, A., \& Ventelou, B. (2012). Granger causality between total expenditure on health and GDP in OECD: Evidence from the Toda-Yamamoto approach. Economics Letters, 116, 541-544. https://doi.org/10.1016/j.econlet.2012.04.040

[4] Aterido, R., Hallaward-Driemeier, M., \& Pagés, C. (2011). Does Expanding Health Insurance Beyond Formal-Sector Workers Encourage Informality?: Measuring the Impact of Mexico's Seguro Popular (N. ${ }^{\circ}$ 5996; IZA Discussion Papers).

[5] Bai, J., \& Carrion-i-Silvestre, J. L. (2009). Structural changes, common stochastic trends, and unit roots in panel data. Review of Economic Studies, 76(2), 471501.

[6] Bai, J., \& Perron, P. (1998). Estimating and Testing Linear Models with Multiple Structural Changes. Econometrica, 66(1), 47-78.

[7] Baltagi, B. H. (2005). Econometric Analysis of Panel Data (3a. ed). John Wiley \& Sons, Ltd.

[8] Baltagi, B. H., \& Moscone, F. (2010). Health care expenditure and income in the OECD reconsidered: Evidence from panel data. Economic Modelling, 27(4), 804-811. https://doi.org/10.1016/j.econmod.2009.12.001

[9] Barro, R. (1996). Health and Economic Growth. World Health Organization.

[10] Becker, G. S. (1962). Investment in Human Capital: A Theoretical Analysis. The Journal of Political Economy, 70(5), 9-49. 
[11] Bedir, S. (2016). Healthcare Expenditure and Economic Growth in Developing Countries. Advances in Economics and Business, 4(2), 76-86. https://doi.org/10.13189/aeb.2016.040202

[12] Bleakley, H. (2010). Health, Human Capital, and Development. Annual Review of Economics, 2(1), 283-310.

[13] Bloom, D. E., Canning, D., \& Sevilla, J. (2001). The Effect of Health on Economic Growth: Theory and Evidence (N. ${ }^{\circ} 8587$; NBER Working Paper). http://www.nber.org/papers/w8587

[14] Bloom, D. E., Canning, D., \& Sevilla, J. (2004). The effect of health on economic growth: A production function approach. World Development, 32(1), 1-13. https://doi.org/10.1016/j.worlddev.2003.07.002

[15] Bloom, D. E., Prettner, K., \& Bloom, D. E. (2018). Health and Economic Growth. IZA Discussion Paper, No. 11939.

[16] Breitung, J., \& Pesaran, M. H. (2005). Unit Roots and Cointegration in Panels (N. ${ }^{\circ}$ 1565).

[17] Busso, M., Fazio, M. V., \& Levy, S. (2012). (In) Formal and (Un) Productive: The Productivity Costs of Excessive Informality in Mexico (IDB-WP-341; IDB Working Paper Series).

[18] Comisión Mexicana sobre Macroeconomía y Salud. (2006). Macroeconomía y salud. Invertir en salud para el desarrollo económico. México: Fondo de Cultura Económica.

[19] De la Torre García, R., Rodríguez García, C., Mier, C., Praz, P. E., \& Ramírez, A. P. (2015). Índice de Desarrollo Humano para las entidades federativas, México 2015. Avance continuo, diferencias persistentes. Programa de las Naciones Unidas para el Desarrollo.

[20] Dincer, H., \& Yüksel, S. (2019). Identifying the Causality Relationship between Health Expenditure and Economic Growth: An Application on E7 Countries. Journal of Health System and Policies, 1, 5-23.

[21] Durán-Arenas, L. (2012). Modelo institucional de atención a la salud en México. En Los determinantes sociales de la salud en México (1era. Ed.). México: Fondo de Cultura Económica.

[22] Enders, W. (2015). Applied econometric time series (4a. Ed). John Wiley \& Sons.

[23] Engle, R. F., \& Granger, C. W. J. (1987). Co-Integration and Error Correction: Representation, Estimation, and Testing. Econometrica, 55(2), 251-276.

[24] Fuentes-Castillo, M. E. (2016). La relación de largo plazo entre salud y crecimiento económico en México y sus entidades federativas, 1940-2011. (Tesis doctoral). Universidad Autonoma de Coahuila.

[25] Gerdtham, U. G., \& Jönsson, B. (2000). International comparisons of health expenditure: Theory, data and econometric analysis. En Handbook of Health Economics (Vol. 1, pp. 11-53). Elsevier.

[26] Gerdtham, U. G., \& Löthgren, M. (2000). On stationarity and cointegration of international health expenditure and GDP. Journal of Health Economics, 19, 461-475.

[27] Gómez Fröde, C. (2017). El sistema de cuentas nacionales de salud en México. Revista CONAMED, 22(3), 129-135.

[28] Granger, C. J. W. (1969). Investigating Causal Relations by Econometric Models and Cross-spectral Methods. Econometrica, 37(3), 424-438.

[29] Grimm, M. (2011). Does inequality in health impede economic growth? Oxford Economic Papers, 63(3), 448-474. https://doi.org/10.1093/oep/gpr002 
[30] Grossman, M. (2000). The Human Capital Model. En Handbook of Health Economics (Vol. 1, pp. 347-408).

[31] Hsiao, C. (2007). Panel data analysis. Advantages and challenges. Test, 16, 1-22.

[32] Hsiao, C. (2014). Analysis of panel data: Third edition. En Analysis of Panel Data (Third Edit). Cambridge University Press.

[33] Im, K. S., Pesaran, M. H., \& Shin, Y. (2003). Testing for unit roots in heterogeneous panels. Journal of Econometrics, 115(1), 53-74.

[34] Johansen, S. (1991). Estimation and Hypothesis Testing of Cointegration Vectors in Gaussian Vector Autoregressive Models. Econometrica, 59(6), 1551-1580.

[35] Levin, A., Lin, C. F., \& Chu, C. S. J. (2002). Unit root tests in panel data: Asymptotic and finite-sample properties. Journal of Econometrics, 108(1), 124.

[36] Levy, S. (2008). Good Intentions, Bad Outcomes: social policy, informality, and economic growth in Mexico. The Brooking Institution.

[37] Lorenzo-Valdes, A., \& Ruiz-Porras, A. (2017). Producción estatal y tasas de natalidad y mortalidad en México: un análisis de sus relaciones de largo plazo y dinámicas. Acta Universitaria, 27(NE-1), 17-32. https://doi.org/10.15174/au.2017.1498

[38] Maddala, G. S., \& Wu, S. (1999). A Comparative Study of Unit Root Tests with Panel Data and a New Simple Test. Oxford Bulletin of Economics and Statistics, 61(S1), 631-652.

[39] Martínez-Trejo, L. O. (2018). Sistema de Salud en México. Encrucijada, Revista Electrónica del Centro de Estudios en Administración Pública, 29, 1-15.

[40] Mayer-Foulkes, D. (2001). The long-term impact of health on economic growth in Mexico, 1950-1995. Journal of International Development, 13(1), 123-126.

[41] Mayer-Foulkes, D. (2008). The Human Development Trap in Mexico. World Development, 36(5), 775-796. https://doi.org/10.1016/j.worlddev.2007.04.023

[42] Mincer, J. (1981). Human Capital and Economic Growth (N. ${ }^{\circ} 803$; NBER Working Paper).

[43] Mushkin, S. J. (1962). Investment in Human Beings. The Journal of Political Economy, 70(5, Part 2), 129-157.

[44] OCDE. (2016). Estudios de la OCDE sobre los sistemas de salud: Mexico. https://doi.org/10.1787/9789264230491-en

[45] Padilla-Hermida, R., \& Garrido-Noguera, C. (2010). Una propuesta de clasificación de las entidades federativas mexicanas para la gestión de la banca de desarrollo. Análisis Económico, 25(58).

[46] Pedroni, P. (1999). Critical values for cointegration tests in heterogeneous panels with multiple regresors. Oxford Bulletin of Economics and Statistics, 61(S1), 653-670.

[47] Pedroni, P. (2001). Fully Modified OLS for Heregoneous Panels. En Nonstationary Panels, Panel Cointegration, and Dynamic Panels (pp. 93-130). Emerald Group Publishing Limited. https://doi.org/dx.doi.org/10.1016/S07319053(00)15004-2

[48] Pedroni, P. (2002). Fully Modified OLS for Heterogeneous Cointegrated Panels. En B. H. Baltagi (Ed.), Recent Developments in the Econometrics of Panel Data (pp. 425-461). Edward Elgar Publishing Limited. 
[49] Pedroni, P. (2004). Panel cointegration: asymptotic and finite sample properties of pooled time series tests with an application to the PPP hypothesis. Econometric Theory, 20, 597-625.

[50] Pedroni, P. (2019). Panel Cointegration Techniques and Open Challenges. En M. Tsionas (Ed.), Panel Data Econometric:Theory. Elsevier Inc.

[51] Rahman, M. M. (2011). Causal Relationship among Education Expenditure, Health Expenditure and GDP: A Case Study for Bangladesh. International Journal of Economics and Finance, 3(3), 149-159. https://doi.org/10.5539/ijef.v3n3p149

[52] Reeves, A., Basu, S., Mckee, M., Meissner, C., \& Stuckler, D. (2013). Does investment in the health sector promote or inhibit economic growth? Globalization and Health, 9(43), 1-12.

[53] Rivera, B., \& Currais, L. (1999). Economic growth and health: Direct impact or reverse causation? Applied Economics Letters, 6(11), 761-764. https://doi.org/10.1080/135048599352367

[54] Rivera, B., \& Currais, L. (2003). The effect of health investment on growth: A causality analysis. International Advances in Economic Research, 9(4), 312323.

[55] Rodríguez-Benavides, D., \& Mendoza-González, M. (2015). Reexaminando la hipótesis de convergencia a la economía líder regional en México: un análisis de cointegración en panel. Paradigma económico, 7(1), 5-48.

[56] Romer, D. (2012). Advanced Macroeconomics (4a Ed). McGraw Hill.

[57] Ruiz-Porras, A., Aceves-Dávalos, J. C., \& León-Cazares, F. (2017). Fondos de aportaciones, desarrollo estatal y elasticidades de sustitución en México: Los impactos de financiar la educación, la infraestructura y la salud. En A. RuizPorras \& F. Venegas-Martínez (Eds.), Avances en economía financiera y desarrollo económico. Modelos analíticos y estudios cuantitativos (pp. 381413). Universidad de Guadalajara.

[58] Schultz, T. W. (1961). Investment in human capital. American Economic Review, 51(1), 1-17. http://www.jstor.org/stable/1818907

[59] Wang, K. M. (2011). Health care expenditure and economic growth: Quantile panel-type analysis. Economic Modelling, 28, 1536-1549.

[60] Westerlund, J., \& Edgerton, D. L. (2008). A simple test for cointegration in dependent panels with structural breaks. Oxford Bulletin of Economics and Statistics, 70(5), 665-704. https://doi.org/10.1111/j.1468-0084.2008.00513.x

[61] Wooldridge, J. M. (2010). Econometric Analysis of Cross Section and Panel Data ( $2 \mathrm{a}$ ed.). The MIT Press. 
